\title{
Methyl Group Relaxations in the Glassy Phase of Polymers
}

\author{
Yoshikazu Tanabe, Juichi Hirose*, Koji OKano, and Yasaku WadA \\ Department of Applied Physics, Faculty of Engineering, \\ University of Tokyo, Bunkyo-ku, Tokyo, Japan.
}

(Received September 13, 1969)

\begin{abstract}
Ultrasonic attenuation at $10 \mathrm{MHz}$ is measured for three poly (methacrylate esters) and polypropylene, and the attenuation peak due to motion of the $\alpha$-methyl group is observed for each polymer. Dynamic mechanical relaxations, NMR line narrowing and $T_{1}$ minima due to methyl group motion are classified into four types according to the location of methyl group in the molecule. The dispersion map is analysed according to the theory by Das. The relaxation time of mechanical relaxation is found to obey the classical theory which does not include the quantum-mechanical tunnelling effect. Mechanical relaxation is interpreted in terms of energy exchange between the methyl group and the backbone chain. Relaxation strength is proportional to absolute temperature. The results of a theoretical treatment of relaxation strength agree satisfactorily with those obtained by experiment.

KEY WORDS Methyl Group / Mechanical Relaxation / NMR / Glassy Polymer / Ultrasonics / Polypropylene / Poly (Methacrylate Esters) /
\end{abstract}

Motion of methyl groups in the glassy phase of polymers has been widely studied by NMR techniques ${ }^{1-4}$. In contrast to NMR experiments, mechanical loss peaks associated with the motion of the methyl group have been observed by some authors ${ }^{5,6}$ but missed by others? and a definite conclusion has not yet been reached whether motion of the methyl group results in dynamic mechanical relaxation or not. In this paper mechanical relaxations are studied by ultrasonic waves for three poly (methacrylate esters) and polypropylene in order to settle this problem. Ultrasonic waves of $10 \mathrm{MHz}$, which have a similar frequency to NMR experiments, are used so that comparison of results obtained with existing NMR $T_{1}$ minimum data may be straightforward.

According to the existing data, temperature positions of $T_{1}$ minimum, line-width narrowing of NMR absorption spectra and dynamic mechanical loss associated with methyl group motion depend appreciably on the chemical structure of the monomeric unit to which the methyl group is attached. In this paper, methyl group relaxations of polymers are classified into

\footnotetext{
* On leave from Tomoegawa Paper Manufacturing Co., Mochimune, Shizuoka, Japan.
}

four types according to the chemical structure of a chain segment adjacent to the methyl group. The temperatures as a function of frequency for each type of methyl group relaxation will be discussed by using the theory by $\operatorname{Das}^{8}$ who calculated the rotational transition frequency of methyl groups over three-fold periodic potential, taking into account the quantum-mechanical tunnelling effect.

The third purpose of this paper is to obtain the temperature dependence of relaxation strength of methyl group relaxation and to discuss it on the basis of statistical mechanical treatment of thermal motion of methyl groups.

\section{CLASSIFICATION OF METHYL GROUP RELAXATIONS}

Temperatures of $T_{1}$ minimum due to the methyl group motion in existing literature ${ }^{1-4}$ are compiled in Table I. The temperature greatly depends on the chemical structure of a segment adjacent to the methyl group. The relaxations are classified into four types according to the methyl group location as shown in Table II.

The A-type relaxation is associated with the ester methyl group and occurs at the lowest 
Table I. Temperatures of minimum spin-lattice relaxation time $\left({ }^{\circ} \mathrm{K}\right)^{\mathrm{a}}$

\begin{tabular}{|c|c|c|c|c|}
\hline Polymer & A & B & $\mathrm{C}$ & $\mathrm{D}$ \\
\hline & $90(50)$ & & & $270(50)$ \\
\hline Poly (methyl methacrylate) & $<70(21)$ & - & - & $260(21)$ \\
\hline Poly (ethyl methacrylate) & - & $130(20)$ & - & $260(20)$ \\
\hline Poly ( $n$-butyl methacrylate) & - & $130(20)$ & - & $260(20)$ \\
\hline Poly ( $n$-lauryl methacrylate) & - & $145(30)$ & - & $245(30)$ \\
\hline Poly ( $n$-docosyl methacrylate) & - & $145(30)$ & - & $250(30)$ \\
\hline Poly (cyclohexyl methacrylate) & - & - & - & $\begin{array}{l}270(50) \\
260(21)\end{array}$ \\
\hline Poly (phenyl methacrylate) & - & - & - & $275(21)$ \\
\hline Poly (methyl acrylate) & $<80(20)$ & - & - & - \\
\hline Poly (ethyl acrylate) & - & $140(20)$ & - & - \\
\hline Poly (methyl $\alpha$-ethacrylate) & - & $120(21)$ & - & - \\
\hline Poly (methyl $\alpha$-chloroacrylate) & $<70(21)$ & - & - & - \\
\hline Poly (methyl $\alpha$-fluoroacrylate) & $90(50)$ & - & - & - \\
\hline Poly (ethyl $\alpha$-chloroacrylate) & - & $140(21)$ & - & - \\
\hline Polypentene-1 & - & $160(50)$ & - & - \\
\hline Polyhexene-1 & - & $150(50)$ & - & - \\
\hline Polyheptene-1 & - & $\begin{array}{l}140(20) \\
150(50)\end{array}$ & - & - \\
\hline \multirow[t]{2}{*}{ Polypropylene } & & & $\begin{array}{l}175(50) \\
165(30)\end{array}$ & \\
\hline & - & - & $160(21)$ & - \\
\hline Polyisobutylene & - & - & - & $240(30)$ \\
\hline Natural rubber & - & - & $160(30)$ & - \\
\hline
\end{tabular}

a Figures in parentheses represent frequency $(\mathrm{MHz})$

Table II. Classification of methyl group relaxation

\begin{tabular}{|c|c|c|c|}
\hline Type & $\begin{array}{l}\text { Location of } \\
\text { methyl group }\end{array}$ & $\begin{array}{c}T_{1} \text { minimum } \\
\text { temperature } \\
\left({ }^{\circ} \mathrm{K}\right) \text { at } \\
50 \mathrm{MHz}\end{array}$ & $\begin{array}{c}\text { Potential } \\
\text { barrier } \\
\text { (kcal/ } \\
\text { mol) }\end{array}$ \\
\hline A & $-\mathrm{O}-\mathrm{Me}$ & 90 & 2.4 \\
\hline B & $\begin{array}{c}-\mathrm{CH}_{2}-\mathrm{Me} \\
\text { C }\end{array}$ & 150 & 3.9 \\
\hline $\mathrm{C}$ & $\begin{array}{c}\text { H- } \\
\stackrel{\text { C }}{\perp}-\mathrm{Me} \\
\mathrm{C} \\
1 \\
\vdots \\
\mathrm{C}\end{array}$ & 190 & 4.4 \\
\hline $\mathrm{D}$ & $\underset{\substack{\mathrm{C} \\
\mathrm{C}}}{\stackrel{\mathrm{C}}{\mathrm{I}}-\mathrm{Me}}$ & 270 & 6.4 \\
\hline
\end{tabular}

temperature, $90^{\circ} \mathrm{K}$ at $50 \mathrm{MHz}$, because of the lowest hindering potential barrier. The B-type relaxation for the methyl group at the end of alkyl group is observed in $130^{\circ}$ to $140^{\circ} \mathrm{K}$ at $20 \mathrm{MHz}$. The C-type relaxation for the $\alpha$-methyl group attached to a tertiary carbon in the backbone chain takes place around $160^{\circ} \mathrm{K}$ at $20 \mathrm{MHz}$. The
D-type relaxation for the $\alpha$-methyl group attached to a quaternary carbon in the backbone chain is observed at the highest temperature, $240^{\circ}$ to $260^{\circ} \mathrm{K}$ at $20 \mathrm{MHz}$.

\section{EXPERIMENTAL}

Velocity and attenuation of ultrasonic longitudinal waves at $10 \mathrm{MHz}$ were measured as a function of temperature by the conventional pulse technique. A schematic diagram of the apparatus is illustrated in Figure 1.

The advantages of high frequency measurements are: (1) the loss peak appears at a high temperature, (2) the accuracy of measurement is high and (3) a sample of small size can be employed and temperature control is easy to maintain.

Two acoustic lines were used, $\mathrm{A}$ the specimen line and $\mathbf{B}$ the reference one. The line $\mathrm{A}$ consisted of two fused silica rods, each $5 \mathrm{~cm}$ long and $2 \mathrm{~cm}$ in diameter, and the specimen was sandwiched 


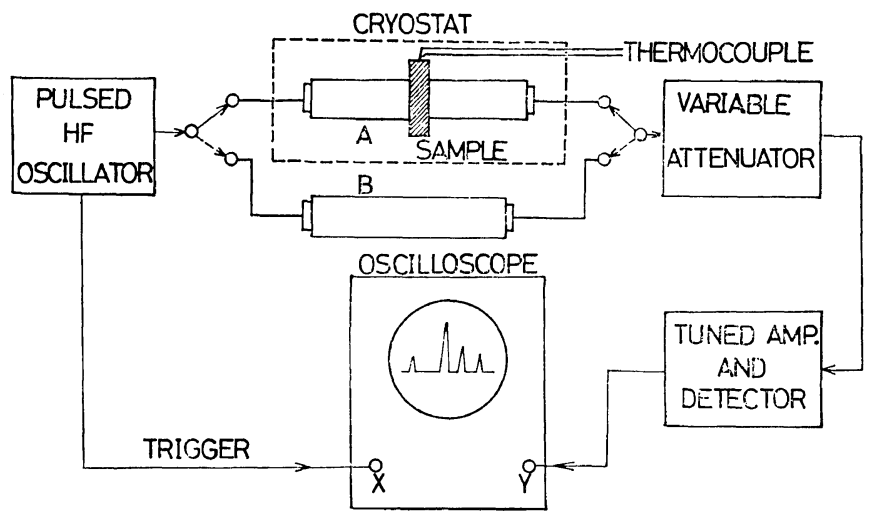

Figure 1. Schematic diagram of ultrasonic apparatus.

between them by use of silicone grease. The thickness of the specimen ranged from 0.5 to $1 \mathrm{~cm}$. The line B consisted of a fused silica rod of $10 \mathrm{~cm}$ in length. The duration of the pulse was so short that interference effect of multiple reflections in the specimen did not occur in the first received signal.

The velocity $c$ in the specimen was obtained from the relationship,

$$
c=l / t
$$

where $l$ is the specimen thickness and $t$ the time difference of received signals between A and B observed on the oscilloscope screen.

The absorption coefficient $\alpha$ in the specimen was obtained by

$$
\alpha=\frac{R-R_{0}}{l}(\mathrm{db} / \mathrm{cm})
$$

where $R(\mathrm{db})$ is the difference in received pulse heights between $\mathrm{A}$ and $\mathrm{B}$ measured with the variable attenuator of $0.1 \mathrm{db}$ step, and $R_{0}$ the reflection loss at the two interfaces in the $\mathrm{A}$ line calculated from acoustic impedances of specimen and fused silica.

The line A was set in a cryostat. The temperature of the specimen was measured with a Cu-constantan thermocouple fixed to it. The measurement was made with temperature increasing from $-120^{\circ} \mathrm{C}$ to room temperature.

Poly (methyl methacrylate) (PMMA), poly (ethyl methacrylate) (PEMA), and poly (iso-butyl methacrylate) (PIBMA) were bulkpolymerized from purified monomers, respectively, with benzoyl peroxide as a catalyst. A commercial, as received sample of isotactic polypropylene (PP) was used.

\section{RESULTS AND DISCUSSION}

Results in Figures 2, 3, and 5, for PMMA, PEMA and PP, respectively, clearly indicate the relaxation peak which should be assigned to methyl group motion by reference to the NMR data. The curve for PIBMA in Figure 4 has a shoulder at $220^{\circ} \mathrm{K}$ besides a major peak at $240^{\circ} \mathrm{K}$. As is well known, PIBMA, which has a long side chain in each monomeric unit, exhibits two sidechain relaxations. The peak at $240^{\circ} \mathrm{K}$ was assigned to the low temperature side-chain relaxation as was confirmed from the data by Hoff et $a l^{9}\left(-150^{\circ} \mathrm{C}\right.$ at $\left.c a .500 \mathrm{~Hz}\right)$, Shen et al. ${ }^{10}$ $\left(155^{\circ} \mathrm{C}\right.$ at $\left.100 \mathrm{~Hz}\right)$ and the transition map of this relaxation $^{11}$, and accordingly the shoulder at

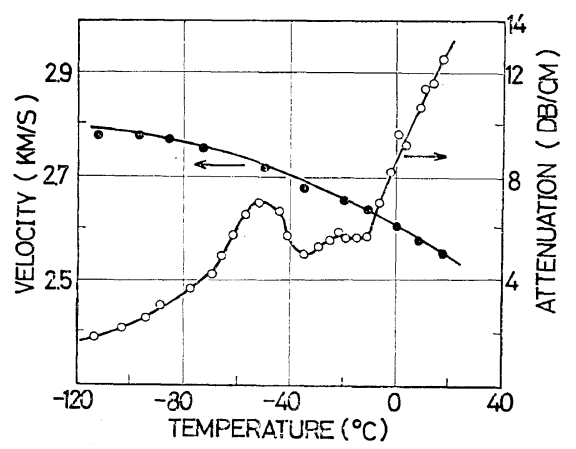

Figure 2. Velocity and attenuation coefficient of longitudinal ultrasonic waves at $10 \mathrm{MHz}$ in poly (methyl methacrylate). 


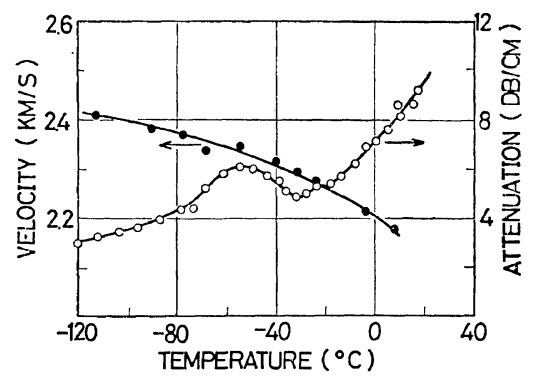

Figure 3. Velocity and attenuation coefficient of longitudinal ultrasonic waves at $10 \mathrm{MHz}$ in poly (ethyl methacrylate).

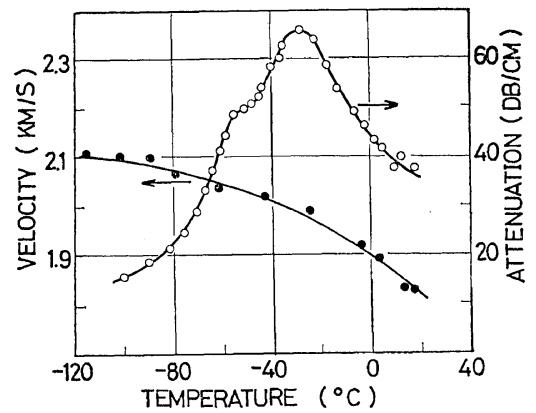

Figure 4. Velocity and attenuation coefficient of longitudinal ultrasonic waves at $10 \mathrm{MHz}$ in poly (isobutyl methacrylate).

$220^{\circ} \mathrm{K}$ was assigned to the methyl peak.

These data show that the methyl group rotation is observed in dynamic mechanical relaxation as well as NMR absorption.

In the following, we will consider why the methyl group rotation results in dynamic mechanical relaxation. At thermal equilibrium, the fluctuation of angular displacement of a methyl group takes an equilibrium distribution. The thermal fluctuation of a methyl group occurs under a three-fold periodic potential, a major part of which may be determined by neighboring atoms in the molecule to which the methyl group is attached (intramolecular potential) but a remaining, minor part may come from atoms in a nearest neighbor chain (or chains) (intermolecular potential). When the specimen is suddenly strained and the intermolecular distance is changed, the intermolecular potential may be changed at the same time and the distribution of angular

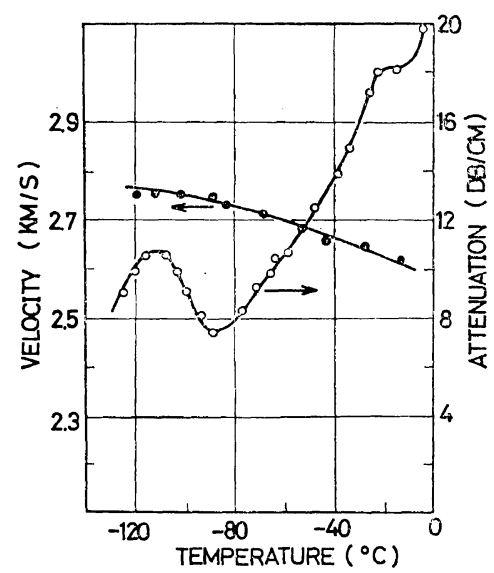

Figure 5. Velocity and attenuation coefficient of longitudinal ultrasonic waves at $10 \mathrm{MHz}$ in polypropylene.

displacement of the methyl group may go to a new equilibrium with a finite relaxation time, accompanying the stress relaxation. Accordingly, the methyl group relaxation is the relaxation of the distribution function of angular displacement of the methyl group, and the energy is irreversibly transfered from the methyl group to the adjacent atoms or vice versa in the course of relaxation.

\section{Relaxation Time}

According to the considerations described in the previous section, the relaxation time depends on the rate of energy transfer between the methyl group and the adjacent segment (the backbone chain in the case of the $\alpha$-methyl group). As a consequence, the relaxation frequency of methyl group relaxation may be the same, in order of magnitude at least, as the transition frequency over the barrier of intramolecular potential,

$$
U_{\text {intra }}=\frac{1}{2} V_{0}(1-\cos 3 \phi)
$$

where $V_{0}$ is the barrier height and $\phi$ the angular displacement. The transition frequency is given by $\operatorname{Das}^{8}$ as

$$
\begin{aligned}
\nu= & \frac{3}{2 \pi}\left(\frac{V_{0}}{2 I}\right)^{1 / 2} \exp \left(-\frac{V_{0}}{k T}\right)+\frac{2 \pi}{9} \\
& \times \frac{\sum_{n} \Delta \nu_{n} \exp \left(-h \nu_{n} / k T\right)}{\sum_{n} \exp \left(-h \nu_{n} / k T\right)}
\end{aligned}
$$


where $I$ is the moment of inertia of the methyl group around the three-fold symmetric axis, $k$ the Boltzmann constant, $h$ the Planck constant, and $T$ the absolute temperature. The first term in the right-hand side of Eq. 4 represents the rate of the transition over the barrier and may be denoted $\nu_{\text {class. }}$ The second term represents the average tunnelling rate. For the three-fold sinusoidal potential of Eq. 3, vibrational energy levels occur in pairs, one of which is degenerate. In Eq. $4 h \nu_{n}$ represents the average energy level of the $n$-th pair $(n=0,1,2, \ldots)$ and $\Delta \nu_{n}$ denotes the splitting of the $n$-th pair, which is a direct measure of the rate of tunnelling through the barrier for the $n$-th state. Both $\nu_{n}$ and $\Delta \nu_{n}$ are expressed in terms of $V_{0}$ and $I$, and the numerical evaluation of $\nu$ has been carried out by Stejskal and Gutowsky ${ }^{12}$.

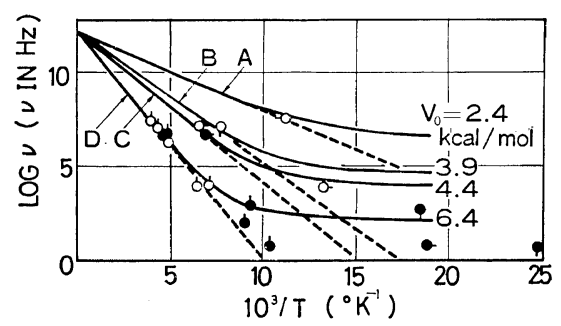

Figure 6. Transition map of four types of methyl group relaxations. $\bigcirc$ : Minimum of spinlattice relaxation time and NMR narrowing. Dynamic mechanical loss peak. (Pip left A-type, pip down B-type, pip right C-type, pip up D-type). - : Quantummechanical theory. -----: Classical theory.

Peak temperatures of dynamic mechanical loss, $T_{1}$ minimum temperatures, and NMR narrowing temperatures for four types of methyl group relaxations are plotted against frequency in Figure 6, by compiling the present data as well as those by other authors. The solid lines in Figure 6 represent theoretical values of Eq. 4, in which $V_{0}$ is chosen to get the best fit with experimental plots at high frequencies. The values of $V_{0}$ thus obtained are listed in Table II, being consistent with the barrier height of internal rotation of small molecules of a similar chemical structure ${ }^{12}$.

The broken lines in Figure 6 show the first term in the right-hand side of Eq. 4, the classical transition frequency over the barrier. From an experimental point of view, one cannot say any- thing conclusive which would be in better agreement with NMR experiments, quantum-mechanical or classical theory, because of a lack of low frequency NMR data. In the case of dynamic mechanical relaxation, however, it is clear that the classical transition frequency agrees with experiment.

As described in the previous section, the dynamic mechanical methyl group relaxation comes from relaxation of distribution in rotational displacement of the methyl group. The intermolecular potential is determined by the position of hydrogen atoms and is not influenced by the rotation of the methyl group by $2 \pi / 3$ due to the tunnelling effect. In the case of NMR absorption, on the other hand, the relaxation is related not only to the position of protons but also to the nuclear spin orientation of the protons, and the rotation due to tunnelling may be effective because three protons in the methyl group may have different spin orientations.

The activation energy obtained from the halfwidth of loss vs. temperature curve in Figure 2 on the assumption of single relaxation mechanism is $5.6 \mathrm{kcal} / \mathrm{mol}$ which roughly agrees with the value from the slope in Figure 6. This indicates that methyl group relaxation is almost a single relaxation process as has been confirmed also from the temperature dependence of $T_{1}{ }^{1}$.

\section{Relaxation Strength}

Figure 7 illustrates the temperature dependence of loss peak height of the D-type relaxation of PMMA. The data were obtained from existing papers $^{5,6}$ as well as the present work. Since the modulus $G$ is almost constant at low temperatures because of the small relaxation strength of

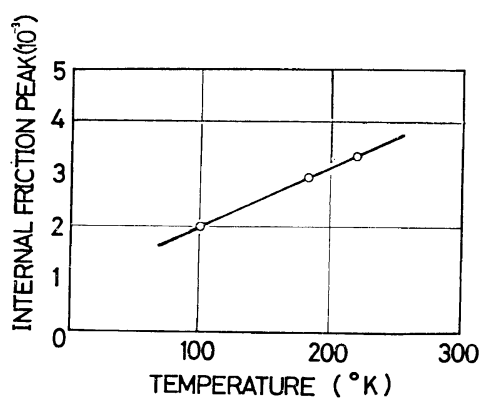

Figure 7. Loss factor peak height plotted against temperature for D-type methyl group relaxation in poly (methyl methacrylate). 
the methyl group and the relaxation is almost a single relaxation type as described in the last section, the peak of internal friction $Q^{-1}$ max is approximately proportional to the relaxation strength $\Delta G$,

$$
\Delta G=2 G Q_{\max }^{-1}
$$

The result in Figure 7 indicates that $\Delta G$ is nearly proportional to the absolute temperaure. Theoretical estimation of the relaxation strength is carried out along the following considerations. When the strain is suddenly applied to the polymer, the intermolecular distance is changed stepwise with time, leaving the rotational state of the methyl group unchanged. The external work is stored, therefore, as an intermolecular potential energy which is equal to $(1 / 2) G(\infty) e^{2}$ per unit volume, where $G(\infty)$ is the instantaneous elastic modulus and $e$ the strain.

Then the statistical distribution of the rotational state of methyl groups goes towards a new equilibrium pertaining to the perturbed potential energy with an appropriate relaxation time $\tau$. The intermolecular energy is irreversibly dissipated finally into thermal motion of the backbone chain to which the methyl group is attached. When the new equilibrium is reached, the stored elastic energy is $(1 / 2) G(0) e^{2}$, where $G(0)$ is the equilibrium elastic modulus. The difference $\Delta G=G(\infty)$ $-G(0)$ represents the relaxation strength.

It follows from the above statement that the motion of the methyl group over the potential barrier is not required for relaxation to occur and only the large amplitude vibration of the methyl group which has an anharmonic coupling with vibrations of the backbone chain is necessary. This implies the preceding discussion of the relaxation time is only the first approximation and the exact calculation of the relaxation time might be obtained from the coupling of vibrations of the methyl group and backbone chain.

To proceed with the calculation of $\Delta G$, the arrangement of methyl groups must be assumed. The model adopted here is illustrated in Figure 8, where a pair of methyl groups is coaxially alligned. This does not mean a pair of methyl groups is necessary for relaxation to occur. Such an arrangement is, however, usually expected in real polymers-isotactic polypropylene in a mono-

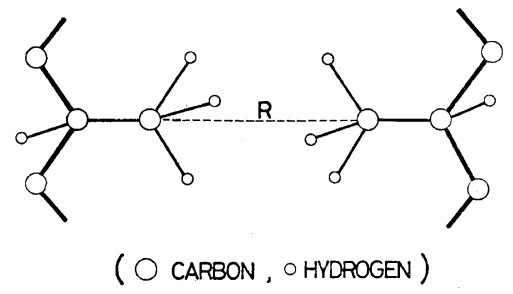

Figure 8. Arrangement of C-type $\alpha$-methyl-group pair.

clinic crystal being an example ${ }^{13}$. It must be noted the arrangement of molecules in the glassy phase may be locally similar to that in the crystal.

The potential energy of this system at equilibrium is written as

$$
\begin{aligned}
U= & \frac{1}{2} V_{0}\left(1-\cos 3 \phi_{1}\right)+\frac{1}{2} V_{0}\left(1-\cos 3 \phi_{2}\right) \\
& +V_{01}+\frac{1}{2} V_{11}\left\{1-\cos 3\left(\phi_{1}-\phi_{2}-\phi_{0}\right)\right\}
\end{aligned}
$$

where $V_{0}$ is the barrier of intramolecular potential, $\phi_{1}$ and $\phi_{2}$ the angle of rotation of each methyl group, $V_{01}$ and $V_{11}$ angle-independent and angledependent parts of intermolecular potential, respectively, and $\phi_{0}$ the angle between equilibrium positions of two methyl groups. Both $V_{01}$ and $V_{11}$ are functions of the distance $R$ between the two methyl groups.

When the system is strained and $R$ is changed, the energy is modified to

$$
\begin{aligned}
U^{\prime}= & \frac{1}{2} V_{0}\left(1-\cos 3 \phi_{1}\right)+\frac{1}{2} V_{0}\left(1-\cos 3 \phi_{2}\right) \\
& +\left(V_{01}+\Delta V_{01}\right)+\frac{1}{2}\left(V_{11}+\Delta V_{11}\right) \\
& \times\left\{1-\cos 3\left(\phi_{1}-\phi_{2}-\phi_{0}\right)\right\}
\end{aligned}
$$

We consider a sinusoidally varying strain with the angular frequency $\omega$. The statistical average of the potential energy of the strained system is

$$
E(0)=\frac{\iint U^{\prime} \exp \left(-\beta U^{\prime}\right) d \phi_{1} d \dot{\phi}_{2}}{\iint \exp \left(-\beta U^{\prime}\right) d \dot{\phi}_{1} d \dot{\phi}_{2}}
$$

and 


$$
E(\infty)=\frac{\iint U^{\prime} \exp (-\beta U) d \phi_{1} d \dot{\phi}_{2}}{\iint \exp (-\beta U) d \phi_{1} d \dot{\phi}_{2}}
$$

where $\beta$ stands for $1 / k T$.

For a small amplitude of strain, $\Delta V_{11} \ll k T$, and hence

$$
\begin{aligned}
\Delta E= & E(\infty)-E(0) \\
= & \frac{\Delta V_{11}}{V_{11} k T}\left[\left(\left\langle U_{0} U_{1}\right\rangle_{0}-\left\langle U_{0}\right\rangle_{0}\left\langle U_{1}\right\rangle_{0}\right)\right. \\
& \left.+\left(\left\langle U_{1}^{2}\right\rangle_{0}-\left\langle U_{1}\right\rangle_{0}^{2}\right)\right]
\end{aligned}
$$

where $\langle\Phi\rangle_{0}$ stands for the average

$$
\langle\Phi\rangle_{0}=\frac{\iint \Phi \exp (-\beta U) d \phi_{1} d \dot{\phi}_{2}}{\iint \exp (-\beta U) d \phi_{1} d \phi_{2}}
$$

and

$$
\begin{aligned}
& U_{0}=\frac{1}{2} V_{0}\left(1-\cos 3 \phi_{1}\right)+\frac{1}{2} V_{0}\left(1-\cos 3 \phi_{2}\right) \\
& U_{1}=\frac{1}{2} V_{11}\left\{1-\cos 3\left(\phi_{1}-\phi_{2}-\phi_{0}\right)\right\}
\end{aligned}
$$

Since the intermolecular, van der Waals potential is much smaller than intramolecular potential, it is safely assumed $V_{11} \ll V_{0}$. This leads to the simple expression (see Appendix),

$$
\begin{aligned}
\Delta E= & \Delta V_{11}\left(\frac{V_{11}}{V_{0}}\right) \sin ^{2} 3 \phi_{0} \\
& +\Delta V_{11}\left(\frac{k T}{V_{0}}\right)\left(\cos 3 \phi_{0}-\frac{3 V_{11}}{V_{0}} \sin ^{2} 3 \phi_{0}\right)
\end{aligned}
$$

On the basis of the symmetry of crystal structure, we assume $\phi_{0}=0$. Then

$$
\Delta E=\Delta V_{11}\left(\frac{k T}{V_{0}}\right)
$$

Since the first derivative of $V_{11}$ with respect to the strain $e=\Delta R / R$ vanishes,

$$
\Delta V_{11}=\frac{1}{2} \frac{\partial^{2} V_{11}}{\partial e^{2}} e^{2}
$$

The relaxation strength is related to $\Delta E$ as follows,

$$
\Delta G=G(\infty)-G(0)=\frac{2 N \Delta E}{e^{2}}=\frac{N k T}{V_{0}}\left(\frac{\partial^{2} V_{11}}{\partial e^{2}}\right)
$$

where $N$ is the number of methyl group pairs per unit volume. Eq. 17 indicates that $\Delta G$ is proportional to the absolute temperature.

The value of $V_{11}$ as a function of $R$ can be calculated under the assumption that the interaction potential between methyl groups is the sum of interatomic potential $u(r)$ which is a function of interatomic distance $r$,

$$
u(r)=-\frac{A}{r^{6}}+B \exp (-C r)
$$

The first term of the right-hand side of eq. (18) represents the London dispersion force and the second term the repulsive force. Numerical values of $A, B$ and $C$ are obtained by de Boer $^{14}$ and Kitaigorodsky ${ }^{15}$ for three species of atom pairs, $\mathrm{H}$ to $\mathrm{H}, \mathrm{H}$ to $\mathrm{C}$, and $\mathrm{C}$ to $\mathrm{C}$.

Calculation for isotactic polypropylene in which $R$ (carbon to carbon distance of methyl groups) is $4.4 \AA$ results in $V_{0}=3 \mathrm{kcal} / \mathrm{mol}$, $\Delta V_{11}=0.23(\Delta R)^{2} \mathrm{kcal} / \mathrm{mol}(\Delta R$ in $\AA)$, and at $160^{\circ} \mathrm{K}, \Delta G=1.8 \times 10^{8} \mathrm{dyn} / \mathrm{cm}^{2}$. As illustrated in Figure 5, the experiment for isotactic polypropylene yields $Q^{-1}{ }_{\max }=4.4 \times 10^{-3}$ at $160^{\circ} \mathrm{K}$, which was obtained by subtracting the background loss from the original data. Since the methyl group relaxation is almost of a single relaxation type, $\Delta G$ is obtained as $1.8 \times 10^{8} \mathrm{dyn} / \mathrm{cm}^{2}$ from Eq. $5\left(G=2 \times 10^{10} \mathrm{dyn} / \mathrm{cm}^{2}\right)$ in a satisfactory agreement with the theoretical value.

In the above theoretical estimation, it was assumed methyl groups in both crystalline and glassy phases contribute to the relaxation and the packing of methyl groups in the crystal is adopted in the glassy phase also. Another simplification is that the theory takes into account only the compressional strain along an axis of a methyl group pair, while in experiment the strain for each methyl group is more complicated.

\section{CONCLUSIONS}

(1) Dynamic mechanical loss peaks due to motion of the $\alpha$-methyl group were observed for polypropylene and three poly (methacrylate esters) by use of an ultrasonic technique at $10 \mathrm{MHz}$, 
and it was concluded that the motion of the methyl group in polymers results in mechanical relaxation as well as NMR narrowing and $T_{1}$ minimum.

(2) Relaxations due to methyl group were classified into four types according to the location of methyl group in the molecule; A-type for ester methyl group, B-type for end methyl group of an alkyl chain, $\mathrm{C}$-type for $\alpha$-methyl group attached to a tertiary carbon, and D-type for $\alpha$-methyl group attached to a quaternary carbon. Each type has a respective relaxation temperature at a given frequency.

(3) The relaxation time and strength of mechanical methyl-group relaxation were theoretically analysed on the basis that the relaxation originates from the change in distribution of angular displacement of the methyl group; the intermolecular elastic energy due to strain is relaxed through energy transfer between the methyl group and the backbone chain.

(4) The relaxation frequency was found to be approximately equal to the transition frequency over the three-fold sinusoidal potential whose barrier height was taken as $2.4,3.9,4.4$, and $6.4 \mathrm{kcal} / \mathrm{mol}$ for $\mathrm{A}$ - to D-types, respectively. The quantum mechanical tunnelling effect was proved ineffective in mechanical relaxation.

(5) A theory for the relaxation strength was developed under the molecular field approximation. The theory predicts the strength is proportional to absolute temperature, which was proved by experiment for the D-type relaxation of poly (methylmethacrylate). Using the arrangement of methyl groups in the crystal of isotactic polypropylene and the numerical parameters of interatomic potentials $(\mathrm{H}$ to $\mathrm{H}, \mathrm{H}$ to $\mathrm{C}$, and $\mathrm{C}$ to C) given by de Boer and Kitaigorodsky, $\Delta G$ (modulus increment from zero frequency to infinitely high frequency) was calculated to be $1.8 \times$ $10^{8} \mathrm{dyn} / \mathrm{cm}^{2}$ at $160^{\circ} \mathrm{K}$ which is in good agreement with experiments for polypropylene.

\section{APPENDIX}

Direct calculation of the average in Eq. 10 is difficult because the interaction term $U_{1}$ is a function of two variables, $\phi_{1}$ and $\phi_{2}$. Derivation of Eq. 14 is, therefore, carried out by separat- ing the variables through molecular field approximation which is useful for simplification of calculation when interaction potential exists.

The average molecular fields for the two methyl groups, $\tilde{U}\left(\phi_{1}\right)$ and $\widetilde{U}\left(\phi_{2}\right)$ are defined by the selfconsistent solution of the following equations,

$$
\begin{aligned}
& \tilde{U}\left(\phi_{1}\right)=\frac{\int \tilde{U}\left(\phi_{1}, \phi_{2}\right) \exp \left\{-\beta \tilde{U}\left(\phi_{2}\right)\right\} d \phi_{2}}{\int \exp \left\{-\beta \tilde{U}\left(\phi_{2}\right)\right\} d \phi_{2}} \\
& \left.\tilde{U}\left(\phi_{2}\right)=\frac{\int U\left(\phi_{1}, \phi_{2}\right) \exp \left\{-\beta \tilde{U}\left(\phi_{1}\right)\right\} d \phi_{1}}{\int \exp \left\{-\beta \tilde{U}\left(\phi_{1}\right)\right\} d \phi_{1}}\right\}
\end{aligned}
$$

Then the average molecular field is obtained from Eqs. 6 and 19 as

$$
\tilde{U}(\phi)=-\frac{1}{2} A \cos 3(\phi-\alpha)+\mathrm{const}
$$

where

$$
\left.\begin{array}{c}
A^{2}=V_{0}^{2}+2 V_{0} V_{11}\left(\eta \cos 3 \phi_{0}-\xi \sin 3 \phi_{0}\right) \\
\tan 3 \alpha=\frac{V_{11}\left(\eta \sin 3 \phi_{0}+\xi \cos 3 \phi_{0}\right)}{V_{0}+V_{11}\left(\eta \cos 3 \phi_{0}-\xi \sin 3 \phi_{0}\right)}
\end{array}\right\}
$$

Parameters $\eta, \xi, \eta^{\prime}$ and $\xi^{\prime}$ are defined by

$$
\left.\begin{array}{rl}
\eta=\langle\cos 3 \phi\rangle, & \xi=\langle\sin 3 \phi\rangle \\
\eta^{\prime}=\langle\cos 6 \phi\rangle, & \xi^{\prime}=\langle\sin 6 \phi\rangle
\end{array}\right\}
$$

where $\langle\Phi\rangle$ stands for the average

$$
\langle\Phi\rangle=\frac{\int \Phi \exp \{-\beta \tilde{U}(\phi)\} d \phi}{\int \exp \{-\beta \tilde{U}(\phi)\} d \phi}
$$

The average appearing in Eq. 10 must be now understood as the average by means of the molecular fields $\widetilde{U}\left(\phi_{1}\right)$ and $\widetilde{U}\left(\phi_{2}\right)$ as shown by Eq. 23 .

In Eqs. 21 and 22, $A / 2$ is the amplitude of the average molecular field and $\alpha$ the setting angle, and $\eta, \xi, \eta^{\prime}$ and $\xi^{\prime}$ represent the degree of the fluctuation of rotation angle of the methyl group and may be called ordering parameters. $\Delta E$ is obtained from replacing $U\left(\phi_{1}, \phi_{2}\right)$ in Eq. 10 by $\left\{\tilde{U}\left(\phi_{1}\right)+\widetilde{U}\left(\phi_{2}\right)\right\}$ 


$$
\begin{aligned}
\Delta E= & \frac{V_{0} \Delta V_{11}}{4 k T}\left[\left\{\left(\eta+\eta \eta^{\prime}+\xi \xi^{\prime}\right)-2 \eta\left(\eta^{2}+\xi^{2}\right)\right\} \cos 3 \phi_{0}\right. \\
& +\frac{V_{11}}{V_{0}}\left\{\frac{1}{2}+\frac{1}{2}\left(\eta^{\prime 2}+\xi^{\prime 2}\right) \cos 6 \phi\right. \\
& \left.\left.-\left(\eta^{2}+\xi^{2}\right)^{2} \cos ^{2} 3 \phi_{0}\right\}\right]
\end{aligned}
$$

Under the assumption $V_{11} / V_{0} \ll 1$, the ordering parameters are obtained by solving simultaneous equations,

$$
\left.\begin{array}{c}
\eta=\cos 3 \alpha \frac{I_{1}(\beta A / 2)}{I_{0}(\beta A / 2)}, \quad \xi=\sin 3 \alpha \frac{I_{1}(\beta A / 2)}{I_{0}(\beta A / 2)} \\
\eta^{\prime}=\cos 6 \alpha \frac{I_{2}(\beta A / 2)}{I_{0}(\beta A / 2)}, \quad \xi^{\prime}=\sin 6 \alpha \frac{I_{2}(\beta A / 2)}{I_{0}(\beta A / 2)}
\end{array}\right\}
$$

where $I_{i}$ is the modified Bessel function of the $i$-th order. Inverse power series expansions of the modified Bessel functions for $\beta V_{0} \gg 1$ are $\left(x=\beta V_{0}\right)$,

$$
\left.\begin{array}{c}
\eta=1-\frac{1}{x}+\frac{1}{x^{2}} \\
\xi=\frac{V_{11}}{V_{0}} \sin 3 \phi_{0}\left(1-\frac{2}{x}+\frac{3}{x^{2}}\right) \\
\eta^{\prime}=1-\frac{4}{x}\left(1-\frac{V_{11}}{V_{0}} \cos 3 \phi_{0}\right) \\
\quad+\frac{4}{x^{2}}\left(1-\frac{3 V_{11}}{V_{0}} \cos 3 \phi_{0}\right) \\
\xi^{\prime}=\frac{2 V_{11}}{V_{0}} \sin 3 \phi_{0}\left(1-\frac{5}{x}+\frac{9}{x^{2}}\right)
\end{array}\right\}
$$

Eq. 24 is simplified to Eq. 14 using the above expansions.

\section{REFERENCES}

1. W. P. Slichter, J. Polym. Sci., Part C, 14, 33 (1966).

2. K. M. Sinnott, J. Polym. Sci., 35, 273 (1959); 3 (1960).

3. J. G. Powles et al., Polymer, 3, 336, 339 (1962); 5 323, 505 (1964).

4. T. Kawai, J. Phys. Soc. Japan, 16, 1220 (1961).

5. K. M. Sinnott, SPE Trans., 2, 65 (1962).

6. P. G. M. Bordoni, Nuovo Cim., 20, 667 (1961).

7. J. M. Crissman et al., J. Polym. Sci., Part A, 2, 5075 (1964); Part A, 3, 2693 (1965).

8. T. P. Das, J. Chem. Phys., 27, 763 (1957).

9. E. A. W. Hoff, D. W. Robinson, and A. H. Willbourn, J. Polymer. Sci., 18, 161 (1955).

10. M. C. Shen, J. D. Strong, and F. J. Matusik, J. Macromol. Sci., B1, 15 (1967).

11. Y. Kawamura, S. Nagai, J. Hirose, and Y. Wada, Reports on Progress in Polymer Physics in Japan, 11, 365 (1968).

12. E. O. Stejskal and H. S. Gutowsky, J. Chem. Phys., 28, 388 (1958).

13. P. H. Geil, Polymer Single Crystals (Interscience, 1963), p. 61.

14. J. de Boer, J. Physica, 9, 363 (1942).

15. A. I. Kitaigorodsky and K. V. Mirskaya, Soviet Phys. Cryst., 9, 137 (1964). 